\title{
Trichosanthin functions as Th2-type adjuvant in induction of allergic airway inflammation
}

\author{
Yuan Wang ${ }^{1}$, Kairui Mao ${ }^{1}$, Shuhui $\mathrm{Sun}^{2}$, Guomei Lin ${ }^{1}$, Xiaodong $\mathrm{Wu}^{1}$, Gang Yao ${ }^{1}$, Bing Sun ${ }^{1,3,4}$ \\ ${ }^{1}$ Laboratory of Molecular Cell Biology, Shanghai Institutes for Biological Sciences, Chinese Academy of Sciences, Shanghai \\ 200031, China; ${ }^{2}$ Laboratory of Molecular Virology, Shanghai Medical School, Fudan University, Shanghai 200032, China; ${ }^{3}$ Insti- \\ tute Pasteur of Shanghai, Chinese Academy of Sciences, Shanghai 200025, China; ${ }^{4}$ E-institutes of Shanghai Universities Immunol- \\ ogy Division, Shanghai 200025, China
}

It is important to understand the pathogenesis of asthma induced by natural allergens, which could exclude the interference of artificial adjuvant and provide insights of natural immune response in the disease. In the present study, we show that Trichosanthin (TCS) could induce airway inflammation even without the help of alum. Furthermore, TCS appeared capable of replacing alum to promote OVA-specific airway inflammation. TCS induced accumulation of IL-4-producing eosinophils in peritoneum at an early stage and the adjuvant function of TCS was eliminated by blockage of IL-4 at this stage. Finally, the eosinophils triggered by TCS from WT mice, but not from IL-4deficient mice were shown to function as adjuvant for the induction of OVA-specific Th2 responses. Our data indicate that TCS is not only an allergen, but also a Th2-type adjuvant modulating the switching of immune responses to a Th2 pathway. This chain of events results from IL-4 production by eosinophils at an early stage of TCS-priming. In conclusion, TCS may be useful as a Th2 adjuvant, and innate immune cells, such as eosinophils, may be a good target to study the initiation of $\mathrm{Th} 2$ response.

Keywords: TCS, Th2, airway inflammation, adjuvant, IL-4, eosinophils

Cell Research (2009) 19:962-972. doi: 10.1038/cr.2009.77; published online 30 June 2009

\section{Introduction}

More than $25 \%$ of the population in industrialized countries suffer from IgE-mediated diseases, one of which is allergic asthma [1]. Various animal models have been established in an attempt to provide insights into the complex immunological and pathophysiological mechanisms of human asthma [2-4]. Of the numerous antigens used in inducing asthma in mice, OVA is the most common one. Due to the interference of alum adjuvant in the OVA-specific immune response in vivo, it is difficult to

Correspondence: Bing Sun ${ }^{\mathrm{a}}$, Gang Yao ${ }^{\mathrm{b}}$

${ }^{a}$ Tel: 86-21-63851927; Fax: 86-21-54921011

E-mail: bsun@sibs.ac.cn

${ }^{b}$ Tel: 86-21-54921012; Fax: 86-21-54921012

E-mail: yaogang@sibs.ac.cn

Abbreviations: TCS (trichosanthin); BALF (bronchoalveolar lavage fluid); MLN (mediastinal lymph node); Ctrl (control); WT (wild type); PEC (peritoneal exudated cell)

Received 18 February 2009; revised 2 March 2009; accepted 4 March 2009; published online 30 June 2009 study the natural immune response to a given allergen in mice. Therefore, it is important to study the mechanism of asthma in an experimental model induced by natural allergens.

Trichosanthin (TCS) is an active component extracted from the root tuber of Trichosanthes kirilowii used in traditional Chinese medicine. It is a $27-\mathrm{kDa}$ protein consisting of 247 amino acid residues. TCS is a member of the type-I ribosome-inactivating protein family and has been used for abortion induction in China $[5,6]$. Since the early 1970s, TCS has been used to terminate early and metaphase pregnancies [7,8]. More recently, TCS was revealed to inhibit HIV replication in infected cells of lymphocytic and mononuclear phagocytic lineages [9, 10]. TCS was also shown to induce anaphylaxis in some treated patients [11]. Indeed, TCS-specific IgE-secreting cells can be detected even 3 years after TCS treatment [12]. Our previous work showed that IgE production increased in TCS-immunized mice [13]. However, the mechanism by which TCS promotes IgE production remains unknown. 
Airway inflammation is a hallmark of asthma, a major public health problem that has increased markedly in prevalence in recent decades [14]. Allergic asthma is a chronic inflammatory disease characterized by pulmonary eosinophilia, increased serum IgE levels, airway hyperreactivity, mucus hypersecretion and structural remodeling of airways [15]. Allergic asthma is driven by inappropriate $\mathrm{Th} 2$-dominated immune responses to environmental allergens. How the immune system is activated by environmental allergens to initiate the Th2 responses is not well understood. Th2 cytokines (IL-4, IL-5 and IL-13 in particular) have been shown to regulate the pathological features of the disease [16-18]. Briefly, IL-4 is critical for priming of B cells and IgE secretion [19]. IL-5 mediates eosinophil recruitment into airways [20]. IL-13 functions in inducing goblet cell hyperplasia, airway remodeling and airway hyperreactivity [21]. Although it has been suggested that IL-4 plays a key role in the initiation of $\mathrm{Th} 2$ response [22], the primary source of IL-4 is poorly understood. Mast cells in humans and eosinophils or basophils in mice have the ability to secret IL-4 in cases of allergic and parasitic worm immunity [23-25]. It has also been reported that IL-4 and IL-13 derived from NKT cells are essential for the development of allergen-induced airway hyperreactivity [26]. However, the source of IL-4 directly induced by natural allergen exposure remains unclear.

Based upon the idea that an animal asthma model induced by a natural allergen would provide an opportunity to study the intrinsic process of this disease, we set up a murine model of airway inflammation using TCS as the allergen. Furthermore, we investigated the contribution of the early IL-4-producing eosinophils triggered by TCS to the induction of airway inflammation.

\section{Results}

TCS can induce allergic airway inflammation in mice in the presence or absence of alum adjuvant

Since TCS exhibits a strong allergenic effect in both humans and mice $[11,13]$, we investigated whether TCS could induce experimental asthma in mice. In our study, C57BL/6 mice were immunized twice with TCS plus alum and challenged with aerosolized TCS. In parallel experiments, PBS and OVA plus alum immunization served as negative and positive controls respectively. The data show that both OVA and TCS could induce allergic airway inflammation in the presence of alum and that the responses were comparable between the two groups based upon the presence of infiltrating inflammatory cells in bronchoalveolar lavage fluid (BALF) (Figure 1A and 1B), antigen-specific IgE production (Figure 1C) and pulmonary histopathology (Figure 1D).

Because TCS can induce strong IgE production in humans without any adjuvant [12], we investigated whether TCS itself could induce allergic responses in mice. Mice were immunized with TCS alone or OVA alone. Following airway challenge, the infiltrating inflammatory cells in BALF of the TCS-treated mice increased significantly as compared with the OVA group (Figure 1E). The numbers of eosinophils in BALF of the TCS group were ten times higher than that of the control group (Figure 1F). In addition, the level of antigen-specific IgE in serum was elevated significantly in the TCS group (Figure 1G). Histological examination also showed that inflammatory responses including inflammatory cell migration, infiltration and accumulation in the airway and perivascular regions of lung occurred in the TCS-sensitized mice, but not in the OVA-sensitized mice (Figure 1H). These observations reveal that administration of TCS induces a severe airway inflammation in the absence of a classical adjuvant. To exclude the possibility of contamination of other molecules, such as endotoxin, we performed high performance liquid chromatography and pyrosate endotoxin detection analyses of the TCS used in the experiments, and confirmed that the reagent used represented a pure single component and was endotoxin free (Supplementary information, Figure S1). Taken together, our results suggest that TCS is a strong allergen and has unique characteristics allowing it to induce allergic responses in mice in the absence of a classic adjuvant.

\section{Th2 response is generated in TCS-treated mice}

The production of Th2 type cytokines, such as IL-4, IL-5 and IL-13 in BALF and mediastinal lymph nodes (MLNs) of TCS-immunized mice was assessed. The data show that the production of IL-4, IL-5 and IL-13 in BALF (Figure 2A) and IL-4 from MLNs (Figure 2B) was significantly increased in TCS-treated mice. Interestingly, the mice immunized with TCS alone appeared to induce higher Th2 responses than those with TCS plus alum immunization. In contrast, the production of IFN- $\gamma$ did not show obvious differences between the groups (Figure 2A and 2B).

To further confirm Th2 responses in vivo, total $\mathrm{IgE}$ and TCS-specific IgG2a isotypes in serum were measured. The data show that the level of IgE was relatively higher in TCS-immunized mice, but the level of $\operatorname{IgG} 2$ a showed no difference between TCS-immunized and control groups (Figure 2C). It was interesting to observe that the TCS plus alum group showed a higher IgG2a level and relatively lower level of $\operatorname{IgE}$ in serum as compared with the group receiving TCS alone. These results indicate that without alum, TCS itself can induce systemic and lo- 

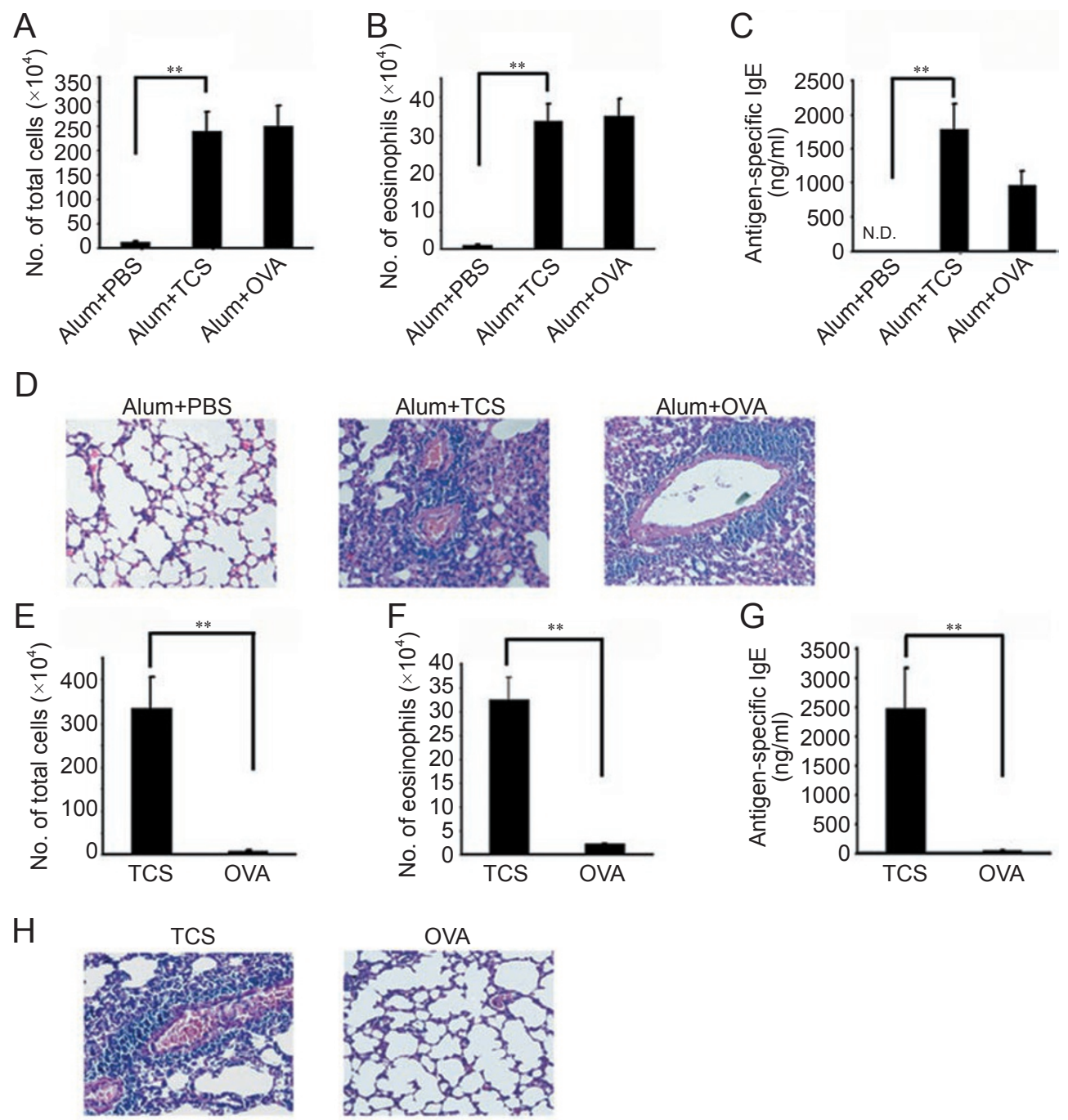

Figure 1 Induction of allergic airway inflammation with TCS. Mice were sensitized with TCS/OVA/PBS as indicated plus alum (A-D) or without alum (E-H) and challenged with aerosolized TCS or OVA or PBS only; $24 \mathrm{~h}$ after the last challenge, mice were sacrificed for analysis. Total infiltrating inflammatory cells ( $\mathbf{A}$ and $\mathbf{E})$ and eosinophils (B and $\mathbf{F})$ in BALF were counted. (C and $\mathbf{G})$ Antigen-specific IgE in serum. (D and $\mathbf{H}$ ) H\&E staining of lung sections. ${ }^{*} P<0.01$.

cal Th2 responses in mice.

TCS functions as Th2 adjuvant to induce OVA-specific allergic airway inflammation

Considering that TCS induces Th2 responses in the absence of alum adjuvant, and our previous work showed that IL-4 gene expression in mesenteric lymph node was increased rapidly after TCS immunization while the IFN- $\gamma$ gene displayed an opposite pattern [27], we asked whether TCS itself has an adjuvant function. Mice were immunized with OVA plus TCS and challenged with aerosolized OVA. Numbers of total infiltrating inflammatory cells and eosinophils in the bronchi increased significantly in mice treated with TCS plus OVA as compared to those treated with OVA alone. The inflammatory responses were comparable between positive control (alum plus OVA) and TCS plus OVA groups (Figure 3A and 3B). OVA-specific IgE was elevated in the TCS plus OVA group (Figure 3C). Histopathology analysis using hematoxylin and eosin (H\&E) staining demonstrated an increase in cell infiltration in TCS plus OVA-treated mice (Figure 3D). Thus, the results indicate that TCS can function as a Th2 adjuvant in inducing OVA-specific allergic responses.

Initial IL-4 production triggered by TCS is critical for TCS-assisted OVA-specific airway inflammation

To determine why TCS has a unique function in promoting an OVA-specific allergic immune response, we investigated whether TCS directly triggered the produc- 
A
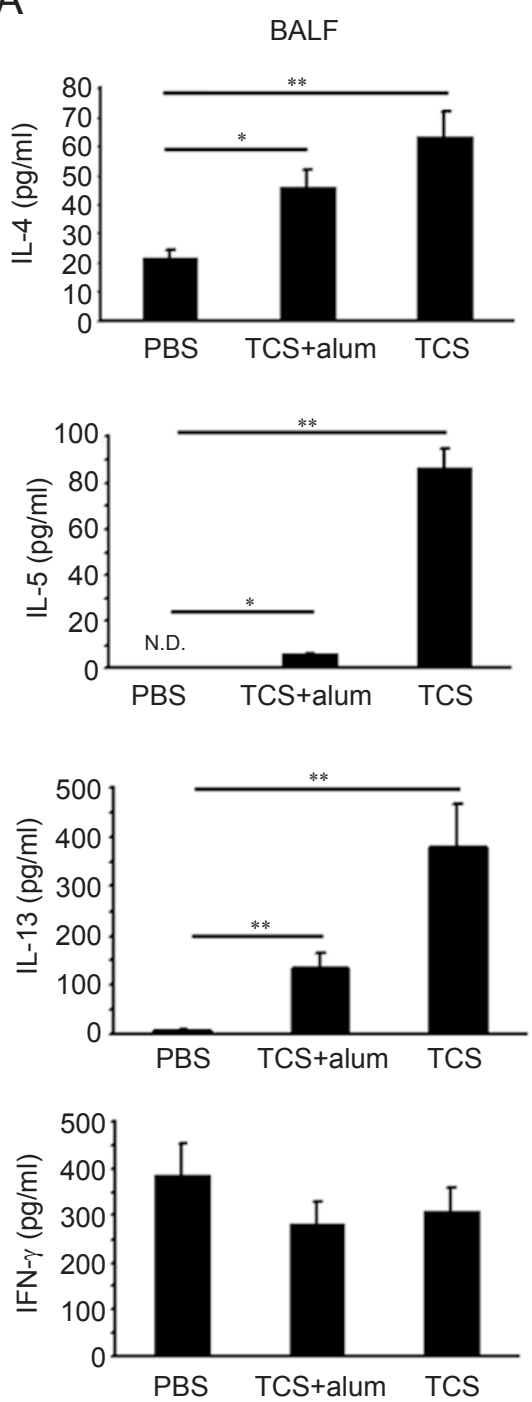

B
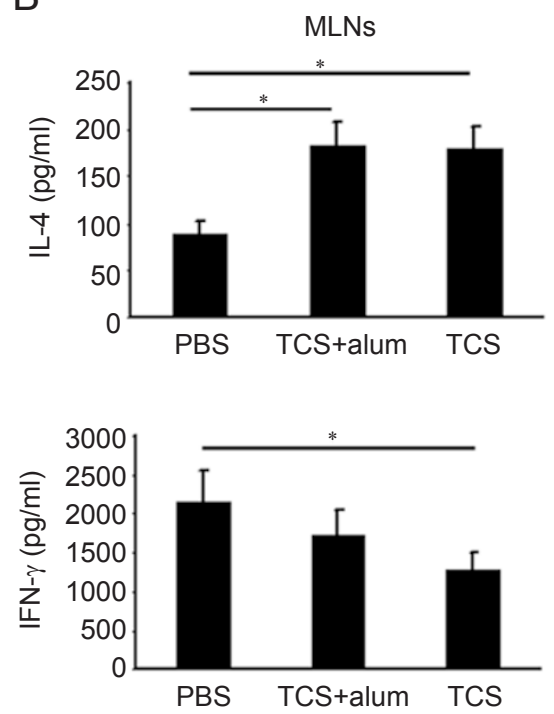

C
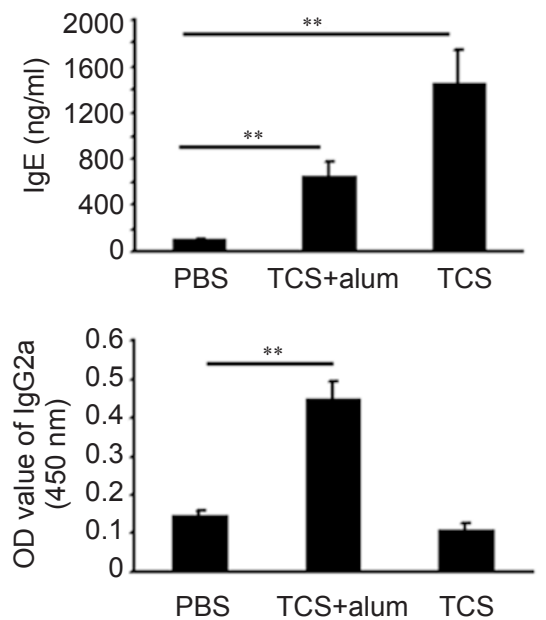

Figure 2 Generation of Th2 responses in TCS-treated mice. Mice were sensitized with TCS plus alum or TCS alone and challenged with aerosolized TCS; $24 \mathrm{~h}$ after the last challenge, mice were sacrificed for analysis. (A) Cytokine production in BALF. (B) Cytokine production in supernatant of culture of MLN cells restimulated with TCS. (C) lg isotypes in serum. $* P<0.05$; $* * P<0.01$.

tion of certain cytokines such as IL-4 or IL-13, thus initiating and favoring the Th2 responses. We found that a single i.p. injection of TCS could induce a rapid increase of systemic IL-4 accumulation in serum, reaching a peak at $12 \mathrm{~h}$ (Figure 4A). We also observed an increase of IL-13 in serum, with a peak at $48 \mathrm{~h}$ (Figure 4B). These results suggest that the initial IL-4 production may create an in vivo environment for Th2-cell differentiation or $\mathrm{B}$ cell activation leading to the pathogenesis of allergic inflammation. To address this hypothesis, the effect of endogenous IL-4 triggered by TCS was abolished by using the neutralizing antibody 11B11. The assay was performed as previously reported [28], and the antidody we used $(<2 \mathrm{mg} /$ mice) would be cleaved in vivo after about 1 day. The data reveal that one injection of $11 \mathrm{~B} 11(0.5$ $\mathrm{mg} / \mathrm{mice}$ ) during TCS immunization could completely block the OVA-specific inflammation as determined by examining the presence of total inflammatory cells and eosinophils in BALF (Figure 4C and 4D), OVA-specific IgE production in serum (Figure 4E) and lung histopathology (Figure 4F). Our study suggests that the initial IL-4 production following TCS immunization is critical for its function as a Th2-type adjuvant.

Eosinophils are identified as the producers of IL-4 at an early stage of TCS priming 

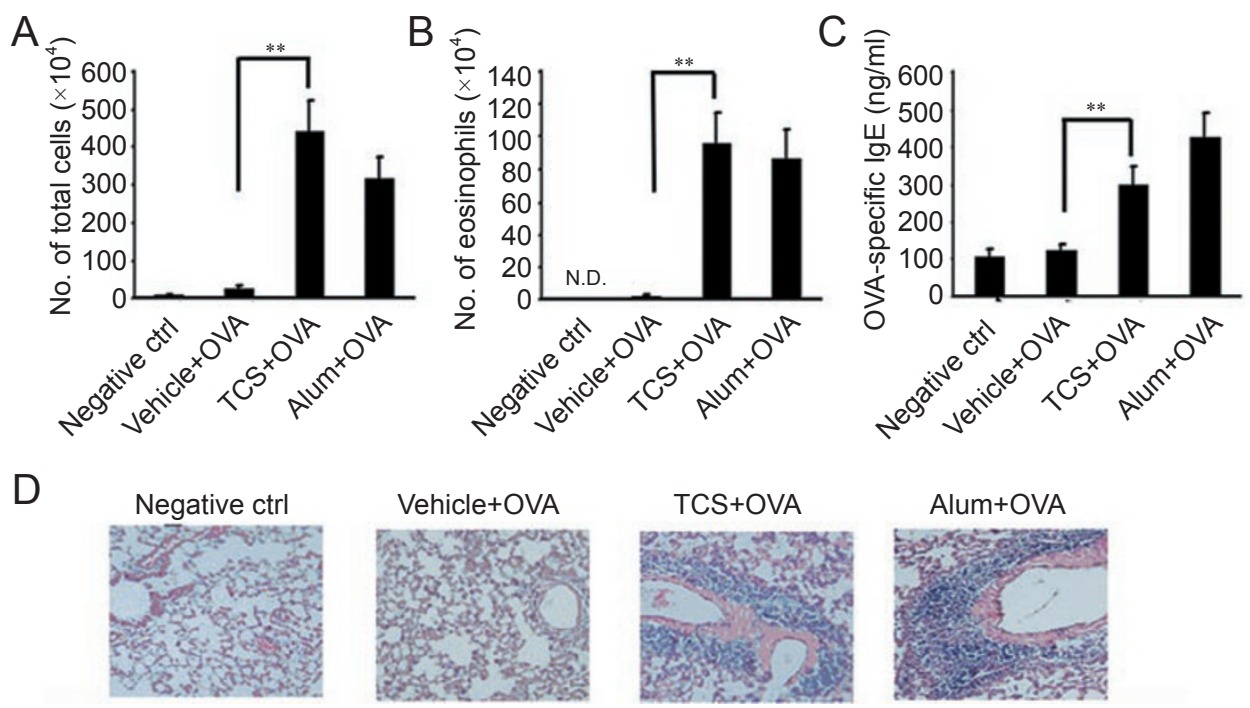

Figure 3 Adjuvant function of TCS in induction of OVA-specific allergic airway inflammation. Mice were immunized with OVA plus TCS or vehicle or alum, and challenged with aerosolized OVA, $24 \mathrm{~h}$ after the last challenge, mice were sacrificed for analysis. Mice immunized and challenged with PBS served as negative control. Total infiltrating inflammatory cells (A) and eosinophils (B) in BALF were counted. (C) OVA-specific IgE in serum. (D) H\&E staining of lung sections. $* * P<0.01$.

A

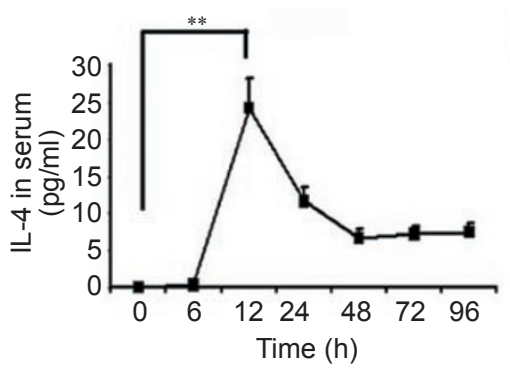

C

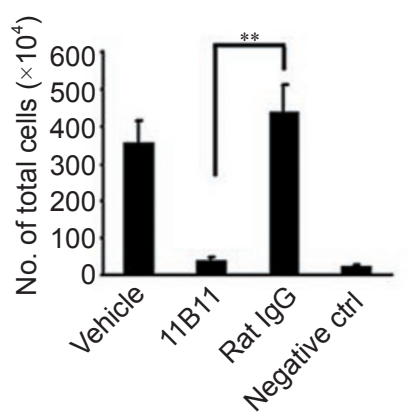

F

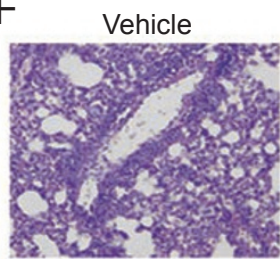

B

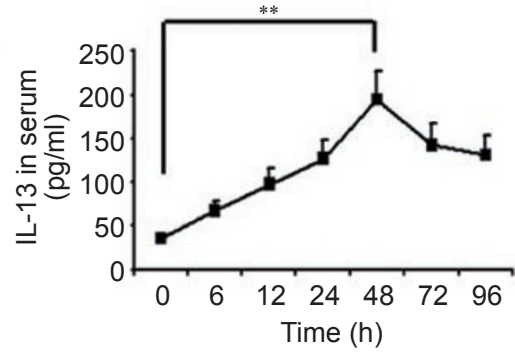

E

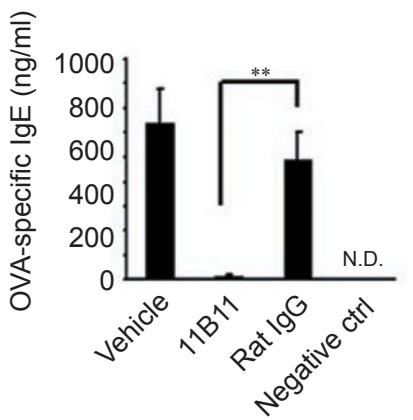

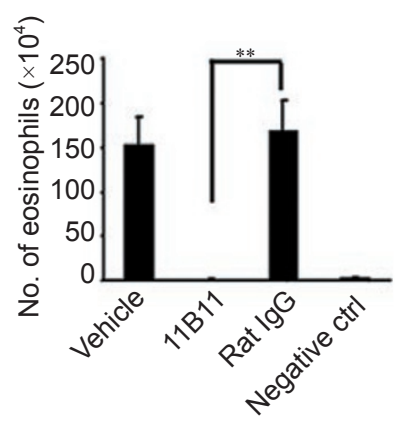

11B11

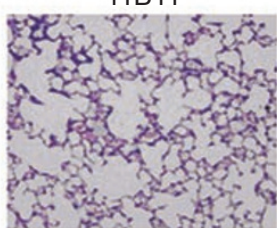

Rat IgG

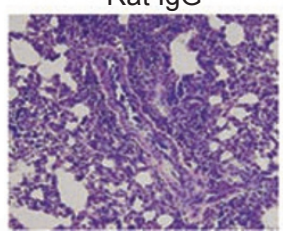

Negative ctrl

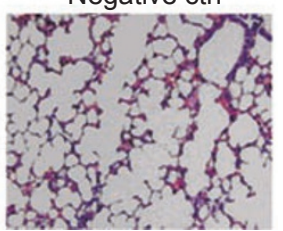

Figure 4 Necessity of early IL-4 production for the development of OVA-specific airway inflammation induced by TCS. IL-4 (A) and IL-13 (B) levels in serum of TCS-treated mice at different time points. (C-F) Mice were immunized with OVA plus TCS. In different groups, mice were treated with 11B11, Rat IgG or vehicle at the same time as TCS injection. Mice were then challenged with aerosolized OVA for five consecutive days. At $24 \mathrm{~h}$ after the last challenge, mice were sacrificed for analysis. Mice immunized and challenged with PBS served as negative control. Total infiltrating inflammatory cells (C) and eosinophils (D) in BALF were counted. (E) OVA-specific IgE in serum. (F) H\&E staining of lung sections. $* * P<0.01$. 
IL-4 production reached a peak at $12 \mathrm{~h}$ following TCS injection, so it is likely that innate immune cells rather than Th2 cells contribute to this IL-4 production. By using 4get mice in which cells that activate the gene encoding IL-4 can be visualized by tracking the expression of eGFP 'knocked into' the $I L-4$ locus [29], we inspected different tissues at a series of time points. We observed that there was a significant increase of a population of IL-4-producing eGFP ${ }^{+}$cells in peritoneal exudated cells (PECs) of mice at $24 \mathrm{~h}$ after TCS injection, but not in OVA-treated mice (Figure 5A). No noticeable IL-4$\mathrm{eGFP}^{+}$cell was found in other tissues (Supplementary information, Figure S2). Further flow cytometry analy- sis showed that the IL-4-eGFP ${ }^{+}$cells had a phenotype

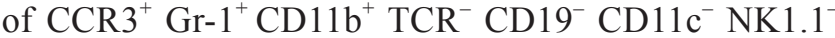

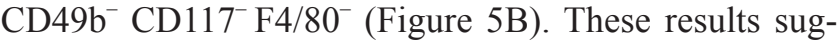
gest that these cells were eosinophils, as CCR3 is considered to be the major marker of eosinophils [30]. To confirm this, we sorted IL-4-eGFP ${ }^{+}$cells and observed their morphological shape by H\&E staining (Figure 5C); the cells $(>99 \%)$ were identified as eosinophils, which is consistent with the result of flow cytometry analysis. Likewise, the absolute numbers of eosinophils in PECs from TCS-immunized C57BL/6 mice are significantly higher than those from PBS-treated group (Supplementary information, Figure S3). TCS thus induces the accumula-

A

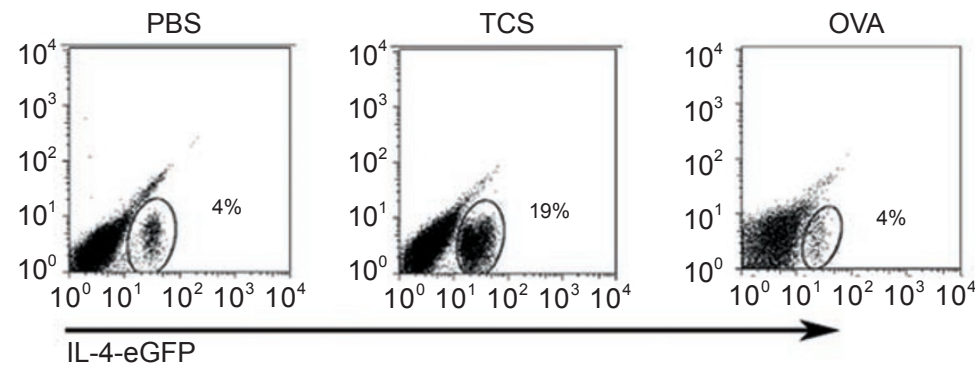

B
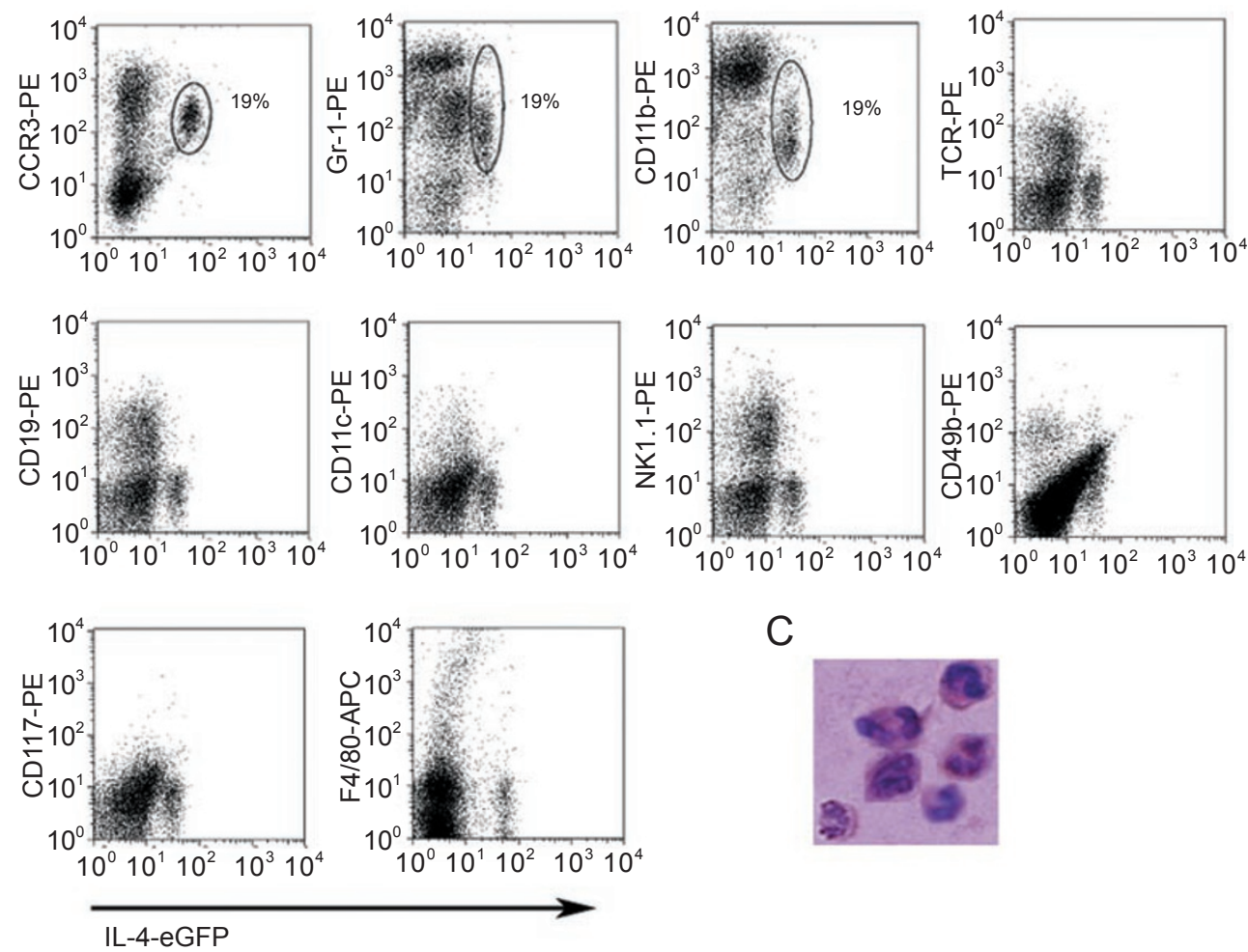

C

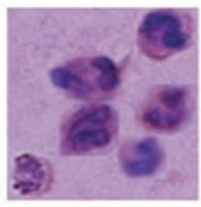

Figure 5 Identification of IL-4 producing cells triggered by TCS. (A) PECs derived from 4get mice immunized with TCS, PBS, or OVA for $24 \mathrm{~h}$ were analyzed by flow cytometry. (B) PECs derived from TCS-immunized 4get mice were stained with the indicated surface marker and analyzed by flow cytometry. (C) H\&E staining of eGFP ${ }^{+}$cells sorted from PECs of TCS-immunized 4get mice. 
tion of IL-4-producing eosinophils in the peritoneum.

Adoptive transfer of TCS-triggered eosinophils favors OVA-specific allergic immune responses

Furthermore, to investigate whether the IL-4-producing eosinophils contribute to the pathogenesis of OVAspecific airway inflammation assisted by TCS, eosinophils were isolated from TCS-immunized WT and $I L-4^{-1-}$ donor mice, and were transferred into WT recipient mice at the same time of OVA immunization on day 1 and day14 without any other adjuvant. The sorting of eosinophils was based on the expression of CCR3 and Gr-1, as previous reported [31]. The purity of the sorted eosinophils was more than $90 \%$, and we confirmed the result by analyzing the morphological shape of these cells using H\&E staining (Supplementary information, Figure S4). Subsequently, mice were challenged with aerosolized
OVA. The significant differences in total inflammatory cells (Figure 6A) and eosinophils (Figure 6B) infiltrating in BALF, levels of OVA-specific IgE in serum (Figure 6C), cytokine production of MLN cells (Figure 6D) and pathology of lung sections (Figure 6E) between groups suggest that eosinophils triggered by TCS could assist OVA to induce airway inflammation, which is dependent upon IL-4 production.

\section{Discussion}

Since almost all available asthma animal models require adjuvant to induce an airway inflammatory response, it has been difficult to identify initial factors that contribute to the pathogenesis of asthma induced by natural allergens. Currently, there is great interest in investigating natural allergens that cause spontaneous
A

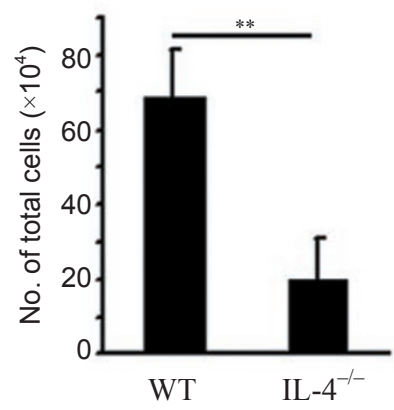

B

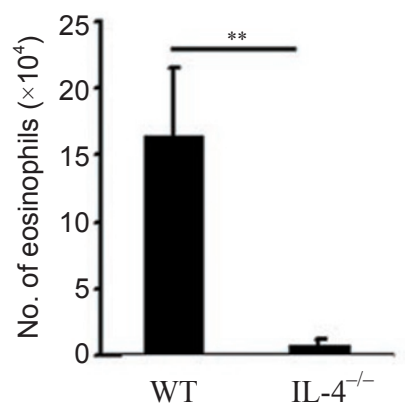

C

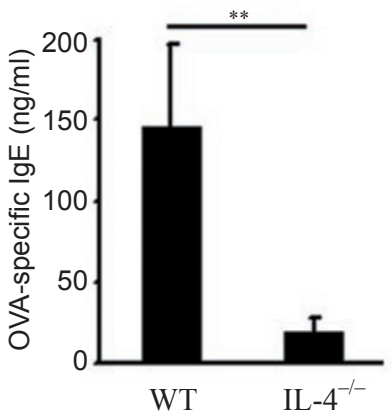

D
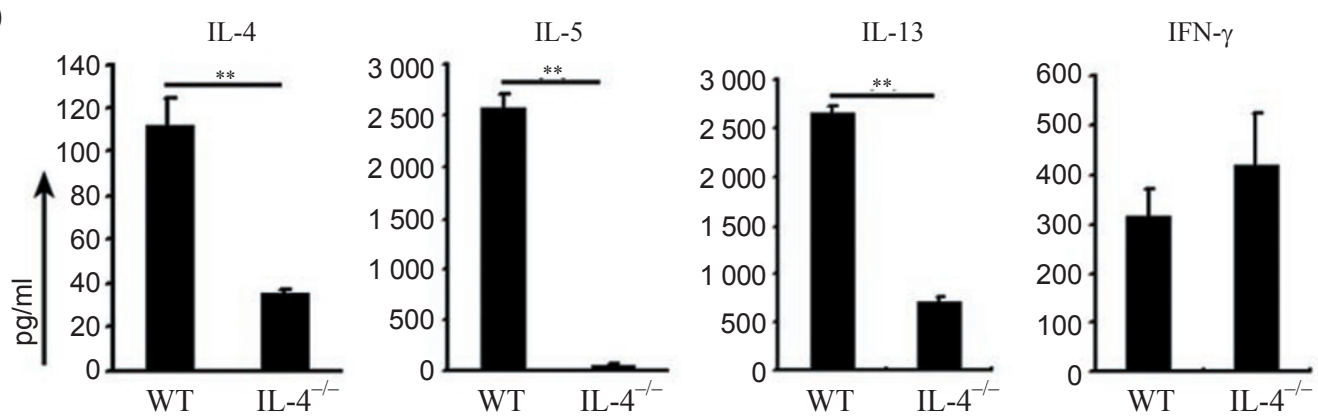

E
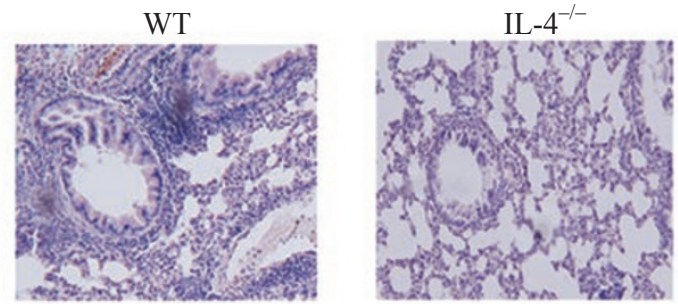

Figure 6 Induction of OVA-specific airway inflammation by adoptive transfer of TCS triggered eosinophils. Eosinophils sorted from PECs of TCS immunized WT or $I L-4^{-/}$mice were transferred to normal mice as while as OVA immunization, and mice were challenged with aerosolized OVA, $24 \mathrm{~h}$ after the last challenge, mice were sacrificed for analysis. Total infiltrating inflammatory cells (A) and eosinophils (B) in BALF were counted. (C) OVA-specific IgE in serum. (D) Cytokine production of MLN cells restimulated with OVA. (E) H\&E staining of lung sections. $* * P<0.01$. 
asthma in humans. These include dust mites [32], pollens (such as ragweed pollens) [33] and protein from dust mites (Derf 1) combined with protein from cockroaches (Bla g 2) [34]. TCS, a single protein [35], has anti-tumor and anti-HIV activities [9, 10]. However, in the clinical application, it can cause IgE production and anaphylaxis in treated patients $[11,12]$. Since TCS appears to be a natural allergen in humans, we attempted to establish a murine airway inflammation model utilizing TCS to investigate the mechanism of the natural process of allergy development.

In this study, we demonstrate that TCS is indeed a strong allergen that induces airway inflammation even without the help of alum adjuvant in mice. In addition, TCS possesses a unique characteristic of Th2-type adjuvant activity that could help in the induction of OVAspecific airway inflammation. Our results suggest that the TCS-induced airway inflammation model is a valuable one for studying the mechanism of asthma in humans.

In general, the Th2-type response is believed to play an important role in the pathogenesis of allergic airway inflammation. In our experiments, we observed that TCS treatment was able to generate a typical $\mathrm{Th} 2$ response. More interestingly, immunization with TCS alone was able to induce airway inflammation and to produce stronger Th2-type responses than those seen with TCS plus alum co-immunization, indicating that TCS itself has a role in initiating Th2-type responses. To further address this hypothesis, we used TCS to replace alum in inducing the OVA-specific airway inflammation. When TCS was injected before OVA injection, allergic airway inflammation occurred following OVA challenge. Thus, TCS could function as a Th2-type adjuvant in promoting OVAspecific airway inflammation and can presumably initiate the Th2 immune response in our animal model.

Further, we investigated the mechanism by which TCS initiates the Th2 responses. As is well known, IL-4 is critical for Th2 cell development and differentiation [36]. We therefore wondered whether TCS could affect IL-4 levels in vivo in mice. The data demonstrate that the IL-4 level in serum was significantly augmented $12 \mathrm{~h}$ after TCS injection. Since IL-13 and IL-4 are at the same gene locus [37], we also analyzed IL-13 in serum. It showed a similar pattern to that of IL-4 after TCS injection. To further confirm the notion that early production of IL-4 triggered by TCS is critical for the function of TCS as an adjuvant, the effect of IL-4 triggered by TCS was abolished by a neutralizing antibody to IL-4 (11B11). We showed that only one injection of $11 \mathrm{~B} 11(0.5 \mathrm{mg} /$ mouse $)$ at the time of TCS immunization could completely block the OVA-specific airway inflammation. This indicates that TCS triggers the early phase IL-4 production, which cre- ates an environment in vivo favoring Th2 cell development and ultimately modulating the immune responses. The cells contributing to this early phase IL-4 production seem not to be $\mathrm{T}$ lymphocytes but rather a population of innate immune cells. Wang et al. [23] reported that the early IL-4 production driving Th2 differentiation in a human in vivo allergic model is derived from mast cells. It was also demonstrated that eosinophils and basophils in mice have the ability to secrete IL-4 under conditions of allergic and parasitic worm immunity [24, 25]; and basophils were assumed to be the initiators of Th2 responses [38]. Besides the cells mentioned above, there are other undefined innate immune cells that could be the early providers of IL-4. It has been found that injection of mice with alum leads to the accumulation in the spleen of a population of IL-4-producing $\mathrm{Gr}-1^{+}$cells, which are required for priming and expansion of antigen-specific $\mathrm{B}$ cells [39]. However, until now, the mechanisms triggering the initial IL-4/IL-13 production have not been very clear. In our present work, we found that at an early stage of TCS injection, a group of IL-4-producing cells accumulated in the peritoneal cavity. We identified these cells as eosinophils. To confirm that these cells could contribute to the pathogenesis of TCS-assisted OVA-specific airway inflammation, we transferred eosinophils from TCStreated WT or $I L-4^{-/-}$mice to WT recipients followed by induction of airway inflammation with OVA. Results show that the eosinophils could replace TCS to favor OVA-specific allergic immune responses, and that their adjuvant function is IL-4 dependent. The results indicate that innate immune cells such as eosinophils triggered by TCS contribute to the initial IL-4 production, thus playing a critical role in initiating the Th2-type response, in favoring B cell priming and in recruiting Th2 cells to lungs as reported recently [31, 40]. Our observations are consistent with the results of Jordan et al. [39] and Wang et al. [41]. Jordan et al. found that injection of mice with alum led to the priming of spleen B cells and to the accumulation of a population of IL-4-producing Gr- $1^{+}$cells in the spleen. Wang et al. identified these cells as eosinophils. However, the mechanism underlying the triggering of eosinophils by TCS or alum remains unclear and needs further investigation. Recently, Trompette et al. [42] showed that Derp 2 has auto-adjuvant properties by interacting with Toll-like receptors. It is certainly possible that natural allergens have common domains or sites, which can be recognized and bound by their specific receptors on immune cells in vivo, resulting in the induction of allergy.

In summary, we demonstrate that TCS has a novel function in inducing Th2-type responses and that TCS itself can induce airway inflammation without alum in- 
volvement. Furthermore, TCS is a Th2-type adjuvant that is capable of replacing alum in inducing OVA-specific allergic responses. More interestingly, this novel function relies upon initial IL-4 production by eosinophils. This indicates that during the natural process of allergic response development, cytokines (such as IL-4) derived from the innate immune cells are critical in initiating a Th2-type response. Our findings provide new insight for the mechanism of Th2-type response initiated by natural allergens, in which the innate immune cells, such as eosinophils, play an important role. Such studies should help provide clues for the development of new strategies in prevention of the allergic diseases. Finally, identification of key molecules and investigation into the function of eosinophils using our model will improve our understanding of the pathogenesis of human asthma.

\section{Materials and Methods}

\section{Animals}

Female C57BL/6 mice, 4get mice and $I L-4^{-/}$mice (6-8 weeks of age) were obtained from the Jackson Laboratory and bred and maintained in the specific pathogen-free animal housing of our Institute. All animal experiments involved groups of 5-10 mice each and were performed according to institutional and state guidelines.

\section{Antigen and reagents}

The crystallized TCS injection solution was purchased from Jin-Shan Pharmaceutical Factory (Shanghai, China). OVA (Grade V) was obtained from Sigma-Aldrich (USA).

Antibodies: Unless especially mentioned, the antibodies used were all purchased from BD Pharmingen (USA).

\section{Induction of allergic airway inflammation in mice}

For induction of TCS-specific airway inflammation, mice were sensitized twice by i.p. with TCS ( $5 \mu \mathrm{g} /$ mouse) in PBS with or without $1 \mathrm{mg}$ alum on days 1 and 14 . From day 21, mice were challenged $30 \mathrm{~min} /$ day with aerosolized TCS $(2 \mathrm{mg} / \mathrm{ml}$ in PBS) for 3 consecutive days. At $24 \mathrm{~h}$ after the last challenge, mice were sacrificed for analysis. For OVA-specific airway inflammation, mice were immunized with OVA (10 $\mu \mathrm{g} /$ mouse $)$ with or without $1 \mathrm{mg}$ alum by i.p. on days 1 and 14. From day 21, mice were challenged $30 \mathrm{~min} /$ day with aerosolized OVA $(1 \% \mathrm{w} / \mathrm{v})$ for 5 consecutive days. $24 \mathrm{~h}$ after the last challenge, mice were sacrificed for analysis. In the TCS-assisted OVA-specific airway inflammation experiments, within $24 \mathrm{~h}$ before OVA immunization, mice were injected by i.p. with TCS ( $5 \mu \mathrm{g} /$ mouse) or vehicle (PBS) as control. In IL4-blocking experiments, $0.5 \mathrm{mg} /$ mouse $11 \mathrm{~B} 11$ or rat $\mathrm{IgG}$ isotype (prepared in our laboratory) or vehicle was injected by i.v. at the same time as the TCS injection.

\section{Collection of bronchoalveolar lavage fluid}

After collection of serum, mice were sacrificed. Lungs were lavaged as previously described [43]. Briefly, the left lung was ligated, the trachea was cannulated and the right lung was lavaged thrice with $500 \mu \mathrm{l}$ PBS. Cell counts were performed on cytospin preparations stained with May-Grünwald-Giemsa (Merck) and at least 200 cells were classified in blinded samples by an independent investigator using standard morphological criteria. Cytokine levels in BALF were determined using commercially available cytokine ELISA kits (Quantikine; R\&D Systems Inc. USA).

\section{Histopathology}

Immediately after collection of lavage fluid, lungs were incised and treated with ice-cold $4 \%$ paraformaldehyde in PBS. Sections of $5 \mu \mathrm{m}$ thickness were cut, mounted on positively charged slides and stained with H\&E.

\section{Serum Ig production}

Serum was separated by centrifugation and stored at $-20{ }^{\circ} \mathrm{C}$ until analysis. TCS and OVA-specific serum IgE, and IgG2a levels were determined using ELISA methodology. HRP-conjugated antimouse IgE (R35-92) or anti-mouse IgG2a (R19-15) was used. For the total IgE analysis, purified anti-mouse IgE capture mAb (R3572 ) was used to coat the plates. Purified mouse IgE (C38-2) served as standard. For IgG2a detection, the serum was diluted 100 times.

\section{Endotoxin detection test}

The tests were performed using the Pyrosate KIT (Catalog number: PSD10). The sensitivity is $0.25 \mathrm{EU} / \mathrm{ml}$. If a solid clot has formed, the result is positive $(+)$. If no clot has formed, the result is negative $(-)$, sample contains $<0.25 \mathrm{EU} / \mathrm{ml}$. TCS injection $(1.2$ $\mathrm{mg} / \mathrm{ml})$ served as sample.

\section{Mediastinal lymph node cytokine secretion}

After mice were sacrificed, MLN cells were isolated, prepared as single cell suspension and seeded on 96-well plates. Cells were restimulated with TCS $(10 \mathrm{ng} / \mathrm{ml})$ or OVA $(50 \mathrm{ng} / \mathrm{ml})$ for $96 \mathrm{~h}$, and the supernatant was collected for cytokine detection using ELISA.

\section{Detection of cytokine levels in serum}

Mice were injected i.p. with $5 \mu \mathrm{g} /$ mouse TCS. At different times after injection, the serum was collected for cytokine detection using ELISA (Quantikine) without dilution.

\section{Flow cytometry and sorting}

PECs were isolated from mice immunized with TCS, OVA or PBS for $24 \mathrm{~h}$. For collection of PECs, peritoneal cavities were lavaged with ice-cold PBS and the lavage fluid was centrifuged and held for further analysis. Total PECs from TCS-treated 4get mice were detected and sorted according to eGFP. For cell type identification, PECs were stained with the following PE-labeled antibodies: anti-mouse CCR3 (83101, R\&D systems), Gr-1 (RB68C5), CD11b (M1/70, eBioscience), TCR (H57-597), CD19 (1D3), CD11c (HL3), NK1.1 (PK136), CD49b (DX-5), CD117 (2B8) and APC-labeled anti-mouse F4/80 (BM8, eBioscence). Isotype controls were conjugated rat IgG2a and hamster IgG. Cells were analyzed using a FACSCalibur cytometer with CellQuest software (BD Biosciences). For sorting eosinophils from TCS-immunized wild-type (WT) and $I L-4^{-/-}$mice, PECs were double stained with FITC-labeled anti-mouse Gr-1 and PE-labeled anti-mouse CCR3.

\section{Eosinophils morphological observation}

Cell counts were performed on cytospin preparations stained with $\mathrm{H} \& \mathrm{E}$ and at least 300 cells were classified using standard morphological criteria. 
Transfer of eosinophils

Eosinophils $\left(3 \times 10^{6} /\right.$ mice $)$ sorted from TCS immunized WT or $I L-4^{-/-}$mice were injected i.p. into C57BL/6 mice at OVA immunization, and followed with induction of airway inflammation with OVA.

\section{Statistics}

For all experiments, the difference between groups was calculated using the unpaired $t$-test. $P$-values less than 0.05 were considered to be significant. Treatment groups of mice consisted of at least five mice each. Experiments were repeated at least thrice. Data shown are representative of these independent experiments; bars represent mean \pm S.E.M.

\section{Acknowledgments}

We thank Prof Yongjun Liu, Dangsheng Li and Yangxin Fu for helpful comments and Dr Sheri Skinner for reviewing the manuscript and for constructive suggestions. This work was supported by grants from the National Natural Science Foundation of China (30530700, 30623003, 30600568, 30721065, 90713044, 30600308, 30801011, 30870126) and CAS project (KSCX1-YW-R-43), grant from SIBS project (2007KIP301), grants from the Ministry of Science and Technology (2006CB504300, 2007CB512404, 2006AA02A247, 20072714), the Technology Commission of Shanghai Municipality (88014199, 07DZ22916, 07XD14033, 064319034, 08431903004, 2008ZX10206, 08DZ2291703), EU project (FP6-2005-SSP-5-B, SP5B-CT-2006-044161) and from the E-institutes of Shanghai Universities Immunology Division.

\section{References}

1 Valenta R. The future of antigen-specific immunotherapy of allergy. Nat Rev Immunol 2002; 2:446-453.

2 Herz U, Renz H, Wiedermann U. Animal models of type I allergy using recombinant allergens. Methods 2004; 32:271280.

3 Kumar RK, Foster PS. Murine model of chronic human asthma. Immunol Cell Biol 2001; 79:141-144.

4 Torres R, Picado C, de Mora F. [Use of the mouse to unravel allergic asthma: a review of the pathogenesis of allergic asthma in mouse models and its similarity to the condition in humans]. Arch Bronconeumol 2005; 41:141-152.

5 May MJ, Hartley MR, Roberts LM, et al. Ribosome inactivation by ricin A chain: a sensitive method to assess the activity of wild-type and mutant polypeptides. EMBO J 1989; 8:301308.

6 Maraganore JM, Joseph M, Bailey MC. Purification and characterization of TCS. Homology to the ricin A chain and implications as to mechanism of abortifacient activity. $\mathrm{J} \mathrm{Biol} \mathrm{Chem}$ 1987; 262:11628-11633.

7 Chang MC, Saksena SK, Lau IF, Wang YH. Induction of midterm abortion by trichosanthin in laboratory animals. Contraception 1979; 19:175-184.

8 Lu PX, Jin YC. Ectopic pregnancy treated with trichosanthin. Clinical analysis of 71 patients. Chin Med J (Engl) 1989; 102:365-367.

9 McGrath MS, Hwang KM, Caldwell SE, et al. GLQ223: an inhibitor of human immunodeficiency virus replication in acutely and chronically infected cells of lymphocyte and mononuclear phagocyte lineage. Proc Natl Acad Sci USA 1989; 86:2844-2848.

10 Gershon D. Compound Q. AIDS drug trial resumes. Nature 1990; 344:183.

11 Jin YC. [Crystal trichosanthin protein intramuscular or intracervical injection for the termination of pregnancy at 10 to 14 weeks gestation: clinical analysis of 200 cases]. Shengzhi Yu Biyun 1990; 10:34-37.

12 Zhuang LQ. [Serum antibody to Radix trichosanthin after its use for termination of midterm pregnancy (author's transl)]. Zhonghua Fu Chan Ke Za Zhi 1979; 14:122-124.

13 Yeh M, Chi YY, Shen RZ, Lin GM. [The study of in vivo and in vitro responses to trichosanthin in the mouse]. Shi Yan Sheng Wu Хue Bao 1986; 19:81-90.

14 Umetsu DT, McIntire JJ, Akbari O, Macaubas C, DeKruyff RH. Asthma: an epidemic of dysregulated immunity. Nat Immunol 2002; 3:715-720.

15 Fahy JV, Corry DB, Boushey HA. Airway inflammation and remodeling in asthma. Curr Opin Pulm Med 2000; 6:15-20.

16 Wills-Karp M. Immunologic basis of antigen-induced airway hyperresponsiveness. Annu Rev Immunol 1999; 17:255-281.

17 Herrick CA, Bottomly K. To respond or not to respond: T cells in allergic asthma. Nat Rev Immunol 2003; 3:405-412.

18 Larche M, Robinson DS, Kay AB. The role of T lymphocytes in the pathogenesis of asthma. J Allergy Clin Immunol 2003; 111:450-463; quiz 464.

19 Hamelmann E, Wahn U, Gelfand EW. Role of the Th2 cytokines in the development of allergen-induced airway inflammation and hyperresponsiveness. Int Arch Allergy Immunol 1999; 118:90-94.

20 Nakajima H, Iwamoto I, Tomoe S, et al. CD4+ T-lymphocytes and interleukin-5 mediate antigen-induced eosinophil infiltration into the mouse trachea. Am Rev Respir Dis 1992; 146:374-377.

21 Wills-Karp M, Luyimbazi J, Xu X, et al. Interleukin-13: central mediator of allergic asthma. Science 1998; 282:22582261.

22 Voehringer D, Reese TA, Huang X, Shinkai K, Locksley RM. Type 2 immunity is controlled by IL-4/IL-13 expression in hematopoietic non-eosinophil cells of the innate immune system. J Exp Med 2006; 203:1435-1446.

23 Wang M, Saxon A, Diaz-Sanchez D. Early IL-4 production driving Th2 differentiation in a human in vivo allergic model is mast cell derived. Clin Immunol 1999; 90:47-54.

24 Sabin EA, Kopf MA, Pearce EJ. Schistosoma mansoni egginduced early IL-4 production is dependent upon IL-5 and eosinophils. J Exp Med 1996; 184:1871-1878.

25 Min B, Prout M, Hu-Li J, et al. Basophils produce IL-4 and accumulate in tissues after infection with a Th2-inducing parasite. J Exp Med 2004; 200:507-517.

26 Akbari O, Stock P, Meyer E, et al. Essential role of NKT cells producing IL-4 and IL-13 in the development of allergeninduced airway hyperreactivity. Nat Med 2003; 9:582-588.

27 Yang CH, Ji YY, Yeh M. The kinetics of IL-4 and IFN-gamma gene expression in mice after Trichosansin immunization. Cell Res 1998; 8:295-302.

28 Yoshino S. Treatment with an anti-IL-4 monoclonal antibody blocks suppression of collagen-induced arthritis in mice by 
oral administration of type II collagen. J Immunol 1998; 160:3067-3071.

29 Mohrs M, Shinkai K, Mohrs K, Locksley RM. Analysis of type 2 immunity in vivo with a bicistronic IL-4 reporter. Immunity 2001; 15:303-311.

30 Heath H, Qin S, Rao P, et al. Chemokine receptor usage by human eosinophils. The importance of CCR3 demonstrated using an antagonistic monoclonal antibody. J Clin Invest 1997; 99:178-184.

31 Jacobsen EA, Ochkur SI, Pero RS, et al. Allergic pulmonary inflammation in mice is dependent on eosinophil-induced recruitment of effector T cells. J Exp Med 2008; 205:699-710.

32 Johnson JR, Wiley RE, Fattouh R, et al. Continuous exposure to house dust mite elicits chronic airway inflammation and structural remodeling. Am J Respir Crit Care Med 2004; 169:378-385.

33 Cates EC, Gajewska BU, Goncharova S, et al. Effect of GMCSF on immune, inflammatory, and clinical responses to ragweed in a novel mouse model of mucosal sensitization. $J$ Allergy Clin Immunol 2003; 111:1076-1086.

34 Sarpong SB, Zhang LY, Kleeberger SR. A novel mouse model of experimental asthma. Int Arch Allergy Immunol 2003; 132:346-354.

$35 \mathrm{Gu} \mathrm{ZF}, \mathrm{Wu} \mathrm{XD}, \mathrm{Li} \mathrm{YJ}$, et al. [Application of trichosanthin in 179 difficult cases of artificial abortion]. Shengzhi Yu Biyun 1985; 5:10-14.

36 Torres KC, Dutra WO, Gollob KJ. Endogenous IL-4 and
IFN-gamma are essential for expression of Th2, but not Th1 cytokine message during the early differentiation of human CD4+ T helper cells. Hum Immunol 2004; 65:1328-1335.

37 Barner M, Mohrs M, Brombacher F, Kopf M. Differences between IL-4R alpha-deficient and IL-4-deficient mice reveal a role for IL-13 in the regulation of Th2 responses. Curr Biol 1998; 8:669-672.

38 Sokol CL, Barton GM, Farr AG, Medzhitov R. A mechanism for the initiation of allergen-induced T helper type 2 responses. Nat Immunol 2008; 9:310-318.

39 Jordan MB, Mills DM, Kappler J, Marrack P, Cambier JC. Promotion of B cell immune responses via an alum-induced myeloid cell population. Science 2004; 304:1808-1810.

40 Walsh ER, Sahu N, Kearley J, et al. Strain-specific requirement for eosinophils in the recruitment of T cells to the lung during the development of allergic asthma. J Exp Med 2008; 205:1285-1292.

41 Wang HB, Weller PF. Pivotal advance: eosinophils mediate early alum adjuvant-elicited B cell priming and IgM production. J Leukoc Biol 2008; 83:817-821.

42 Trompette A, Divanovic S, Visintin A, et al. Allergenicity resulting from functional mimicry of a Toll-like receptor complex protein. Nature 2009; 457:585-588.

43 Heeckeren A, Walenga R, Konstan MW, et al. Excessive inflammatory response of cystic fibrosis mice to bronchopulmonary infection with Pseudomonas aeruginosa. J Clin Invest 1997; 100:2810-2815.

(Supplementary information is linked to the online version of the paper on the Cell Research website.) 